\title{
Selective Suspension in Aqueous Sodium Dodecyl Sulfate According to Electronic Structure Type Allows Simple Separation of Metallic from Semiconducting Single-Walled Carbon Nanotubes
}

\author{
Kai Moshammer ${ }^{1}$, Frank Hennrich ${ }^{1}(\bowtie)$, and Manfred M. Kappes ${ }^{1,2}(\bowtie)$ \\ ${ }^{1}$ Forschungszentrum Karlsruhe, Institut für Nanotechnologie, D-76021 Karlsruhe, Germany \\ ${ }^{2}$ Institut für Physikalische Chemie, Universität Karlsruhe, D-76128 Karlsruhe, Germany \\ Received: 20 April 2009 / Revised: 12 May 2009 / Accepted: 12 May 2009 \\ (C)Tsinghua University Press and Springer-Verlag 2009. This article is published with open access at Springerlink.com
}

\begin{abstract}
Both density gradient centrifugation and gel electrophoresis have been reported to allow high throughput separation of metallic from semiconducting single-walled carbon nanotubes (SWNTs) when using aqueous sodium dodecyl sulphate (SDS) suspensions. We show here that both methods rely on an initial dispersionby-sonication step, which is already selective with respect to electronic structure type. The corresponding aqueous SDS "starting" suspensions obtained after sonication and purification by simple centrifugation $(70,000$ $g, 1 \mathrm{~h}$ ) contain semiconducting SWNTs primarily in the form of small bundles whereas metallic SWNTs are predominantly suspended as individual tubes. Density gradient centrifugation then separates the bundles from the individual tubes on the basis of differences in their overall buoyant densities. Gel electrophoresis separates the longer bundles from the shorter individual tubes on the basis of their different mobilities. We also demonstrate that such starting suspensions can be fractionated according to electronic structure type by even simpler techniques such as size exclusion chromatography or gel filtration, thus opening the way for simple scale-up.
\end{abstract}

\section{KEYWORDS}

Single-walled carbon nanotubes, metallic-semiconducting separation, electrophoresis, density gradient centrifugation, size exclusion chromatography

\section{Introduction}

Development and application of separation techniques for single-walled carbon nanotubes (SWNTs) have become an active research field over the past years. It is driven by the fact that no known synthesis method allows the growth of nanotube samples having preselected diameters and electronic type (metallic (m) or semiconducting (s)). In fact, $\mathrm{m}$-and s-SWNTs are grown together in a complex mixture of many different structure types (described by specific chiral vectors). The "holy grail" of chiral vector-selective synthesis remains elusive in spite of intense efforts to develop appropriate catalysts.

Widespread electronic applications of SWNTs would be greatly facilitated by the routine provision

Address correspondence to Frank Hennrich, Frank.Hennrich@int.fzk.de; Manfred M. Kappes, Manfred.Kappes@chemie.uni-karlsruhe.de 
of samples fractionated by electronic structure type in a simple process. For this reason, separation of $\mathrm{m}$ - from s-SWNTs has become a flourishing subfield and in fact enrichment of $\mathrm{m} / \mathrm{s}$-SWNTs has been achieved by various techniques including (AC) dielectrophoresis [1], density gradient centrifugation [2], and (DC) agarose gel electrophoresis [3]. A recent review article on separation methods in general, including separation of $\mathrm{m}$ - from s- SWNTs, gives a good overview of efforts to date [4].

All such separation methods make use of nanotube/surfactant suspensions in liquids. Most commonly, $\mathrm{H}_{2} \mathrm{O} / \mathrm{D}_{2} \mathrm{O}$ suspensions with surfactants such as sodium dodecyl sulfate (SDS), sodium dodecylbenzene sulfonate (SDBS) or sodium cholate (SChol) have been used. Corresponding separation protocols all start with the preparation of suspensions enriched in "individualized" (i.e., debundled / exfoliated) tubes-typically by means of sonication treatment followed by centrifugation. So far, the focus of published work in this field has been on the demonstration of separation by electronic structure type and more recently on bulk-scale characterization of the resulting enriched samples. Less attention has been paid to the enabling details. In particular, little is known about the dispersion/individualization efficiency and how this might depend on tube type.

In the present contribution we demonstrate that the two methods which have so far been the most promising for bulk-scale separation according to electronic type (gel electrophoresis and density gradient centrifugation), rely on the fact that under the sonication/suspension conditions used, s-SWNTs are individualized less efficiently than m-SWNTs. Density gradient centrifugation (DGC) then separates (mainly semiconducting) bundles from (mainly metallic) individual tubes on the basis of differences in their overall buoyant densities, thus leading to enrichment of metallic tubes in the lower density fraction. DC gel electrophoresis (EP), on the other hand, separates on the basis of different bundle versus individual tube length distributions (and the resulting variations in mobility). As bundles are typically longer than individual tubes, separation by electronic structure type again ensues. We also demonstrate that this new insight into the composition of aqueous SDS suspensions can be used to design an even simpler separation protocol based on size exclusion chromatography (SEC).

\section{Methods}

The SWNT material used for this study was prepared by pulsed laser vaporization (PLV) using carbon targets doped with 1 atom \% Ni and Co catalyst in an argon atmosphere and an oven operated at $1000{ }^{\circ} \mathrm{C}$ [5].

\subsection{Starting suspensions}

Typically $10 \mathrm{mg}$ of SWNT material was suspended in $25 \mathrm{ml} \mathrm{D} \mathrm{D}_{2} \mathrm{O}$ with $1 \mathrm{wt} \%$ of SDS (or SChol for the reference sample) using a tip sonicator (Bandelin, $200 \mathrm{~W}$ maximum power, $20 \mathrm{kHz}$, in pulsed mode with 100 ms pulses) applied for $2 \mathrm{~h}$ at $20 \%$ power. For the EP study, a preliminary DGC step was used to remove larger agglomerates, amorphous carbon, and catalyst particles while retaining SWNTs. The corresponding centrifugation conditions are described in detail in Section 1.3. Self-generated gradients of iodixanol plus $1 \mathrm{wt} \%$ of SChol were typically used. After centrifugation, different coloured regions-approximately $5 \mathrm{~mL}$ of the whole $8 \mathrm{~mL}$ from bottom to top-were visible. Of these, only the last (i.e., topmost) $\sim 0.5 \mathrm{~mL}$ contained pure SWNTs. This last fraction was then isolated and used as the starting suspension for $\mathrm{m} / \mathrm{s}-\mathrm{SWNT}$ separation by EP as described below. For the DGC and the SEC study the sonicated suspension was centrifuged at $\sim 70,000 \mathrm{~g}$ for $1 \mathrm{~h}$ without iodixanol. The resulting supernatant was used then as the starting suspension.

\subsection{Electrophoresis}

EP was performed in a $0.4 \mathrm{wt} \%$ agarose gel filled into a glass pipette $(5 \mathrm{~mm}$ inner diameter, $50 \mathrm{~mm}$ filling height) using a Tris-acetate-EDTA (TAE) buffer plus $1 \mathrm{wt} \%$ SDS at $12 \mathrm{~V}$. After applying $1 \mathrm{~mL}$ of starting suspension and (subsequently) voltage, the SWNTs began to move through the gel and two differently coloured regions characterized by different propagation velocities rapidly became visible ( Fig.1). After $\sim 2 \mathrm{~h}$ the upper part of the agarose gel appeared greenish, whereas the lowermost part exhibited a bluish colour. Different parts of the gel were analysed 


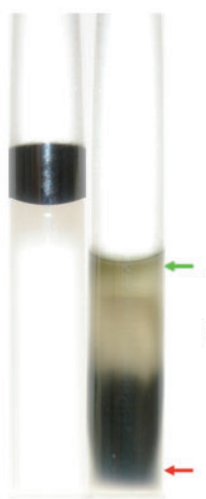

(a)

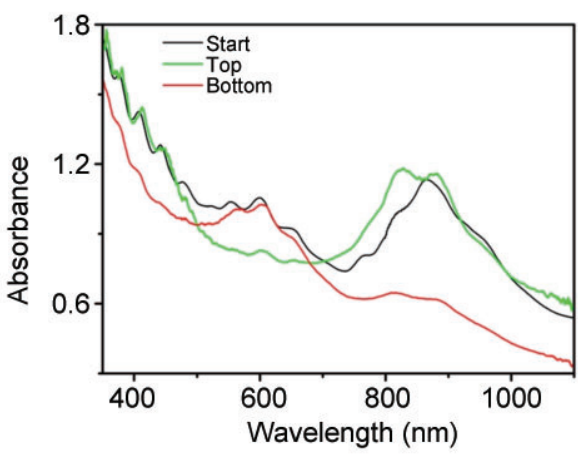

(b)
Figure 1 (a) Photographs taken before and after EP separation of an SWNT starting suspension using an agarose gel; note the appearance of two differently coloured regions after applying a voltage for 2 h; (b) UV-vis-NIR spectra of the starting suspension and spectra measured after EP separation within two different regions of the agarose gel (green: top and red: bottom part of gel)

in situ by UV-vis-NIR absorption spectroscopy using a balanced deuterium tungsten halogen light source (Micropack DH-2000-BAL, Ocean Optics) and a spectrometer detector (HR4000, Ocean Optics) connected via optical fibers to a home built sample holder.

\subsection{Density Gradient Centrifugation}

The DGC protocol used was similar to that of Ref. [2]. However, rather than pre-formed gradients we used self-generated gradients of iodixanol plus $1 \mathrm{wt} \%$ of either SDS or a 3:1 mixture of SDS and SChol. Ultracentrifugation (Optima Max-E, Beckman Coulter) was carried out in a fixed angle rotor (ML-80, Beckman Coulter) at $15{ }^{\circ} \mathrm{C}$ and at 45,000 rpm for 18 $20 \mathrm{~h}$ using polyallomer (8 mL Bell top Quick-Seal, Beckmann Coulter) centrifuge tubes. This rotation speed results in centripetal accelerations of 103,650 $g$ and $140,400 g$, at the average/maximal radii of $45.7 / 61.9 \mathrm{~mm}$, respectively.

In a typical experiment, centrifuge tubes were first filled with $5 \mathrm{~mL}$ of $60 \mathrm{wt} \%$ iodixanol (purchased as OptiPrep, Sigma Aldrich) plus $1 \mathrm{wt} \%$ of SDS or the SDS/SChol mixture. Then, the tube was filled with 3 $\mathrm{mL}$ of the starting nanotube SDS suspension, sealed and centrifuged. In order to harvest the resulting fractions after centrifugation, we punctured small holes in the top and bottom of the centrifuge tubes and forced the liquid through the bottom hole by applying modest excess pressure through the top hole with a small syringe. As a result, the region containing SWNTs was divided into 50 droplet volumes of up to $\sim 25 \mu \mathrm{L}$. These resulting fractions were then diluted with $1 \mathrm{wt} \%$ SDS solution in $\mathrm{D}_{2} \mathrm{O}$ to $0.8 \mathrm{~mL}$ for optical characterization. UV-vis-NIR absorption spectra of the fractions were recorded on a Varian Cary 500 spectrophotometer.

\subsection{Size Exclusion Chromatography}

SEC was performed using Sephacryl S-200 gel filtration medium (Amersham Biosciences) in a glass column of $20 \mathrm{~cm}$ length and $2 \mathrm{~cm}$ inner diameter. After filling the glass column with the filtration medium, the gel was slightly compressed to yield a final height of $14 \mathrm{~cm}$. For the separation, $10 \mathrm{~mL}$ of SWNT starting suspension was applied to the top of the column and a solution of $1 \mathrm{wt} \%$ SDS in $\mathrm{H}_{2} \mathrm{O}$ as eluant was pushed through the column by applying sufficient pressure with compressed air to ensure a flow of $\sim 1 \mathrm{~mL} / \mathrm{min}$. Fractions were collected in 3-4 $\mathrm{mL}$ portions. After $\sim 10 \mathrm{~mL}$ of eluant had been added, the upper part of the gel appeared greenish, whereas the lowermost part exhibited a bluish colour. Even after applying $~ 100 \mathrm{~mL}$ of SDS solution, the greenish NT material stayed within the upper third of the SEC gel whereas the bluish NT eluted completely. By changing the eluant from $1 \mathrm{wt} \%$ SDS in $\mathrm{H}_{2} \mathrm{O}$ to $1 \mathrm{wt} \%$ SChol in $\mathrm{H}_{2} \mathrm{O}$ and applying $\sim 100 \mathrm{~mL}$ of the latter, the greenish part also subsequently eluted completely from the column and was collected separately.

\subsection{Atomic force microscopy}

Atomic force microscopy (AFM) samples were prepared and measured as described in Ref. [6]. Samples were prepared by spin coating of SWNT starting suspensions onto silicon wafers and rinsing with water and acetone. Intermittent Contact Mode AFM images were recorded with a Digital Instruments Multimode SPM with NSC15 silicon cantilevers (MikroMasch). The heights and lengths of the objects measured were extracted from AFM pictures with the help of the Software package SIMAGIS (Smart Imaging Technologies Co.). Tube lengths were determined to within a lateral resolution of $\sim 20 \mathrm{~nm}$. 


\section{Results and discussion}

\subsection{Separation by electrophoresis}

After applying a DC voltage of $12 \mathrm{~V}$ between the upper and lower part of the SWNT-loaded agarose gel (electrode separation $\sim 10 \mathrm{~cm}$ ) for $\sim 2 \mathrm{~h}$, we observed that the upper parts of the gel appeared greenish whereas the lowermost part appeared bluish (Fig. 1(a)). The corresponding absorption spectra taken from different regions within the gel are shown in Fig. 1(b). Compared to the starting suspension, the absorption spectrum of the topmost region showed clear enrichment of s-SWNTs whereas the absorption spectrum of the lowermost region showed clear enrichment of m-SWNTs. In particular, for the metallic fraction, the second interband transition of s-SWNTs in the region between 700 and $1100 \mathrm{~nm}$ was observed to be strongly reduced in intensity. Correspondingly for the semiconducting fraction, the intensities of the first interband transitions of metallic tubes which lie between 500 and $700 \mathrm{~nm}$ were strongly reduced.

Figure 2 provides a rationalization for our observation that EP in an agarose gel allows separation of m-SWNTS from s-SWNTs. Figures 2(a) and 2(b) show AFM-derived length and height distributions for nanotube material spin coated from SDS and SChol (starting) suspensions onto a silicon wafer. The PLV-SWNT material used in both suspensions had an individual nanotube diameter distribution of $1.0 \mathrm{~nm} \pm 0.2 \mathrm{~nm}$. From height analysis of the spin coated deposit we found that a significant fraction of the objects deriving from the SDS suspension had heights considerably larger than 1 $\mathrm{nm}$. This observation was independent of how we generated the starting suspension, i.e., the fraction of bundles as determined by AFM was almost the same for DGC-purified starting suspensions (Fig. 2(c)) as for samples centrifuged at 70,000 $\mathrm{g}$ without

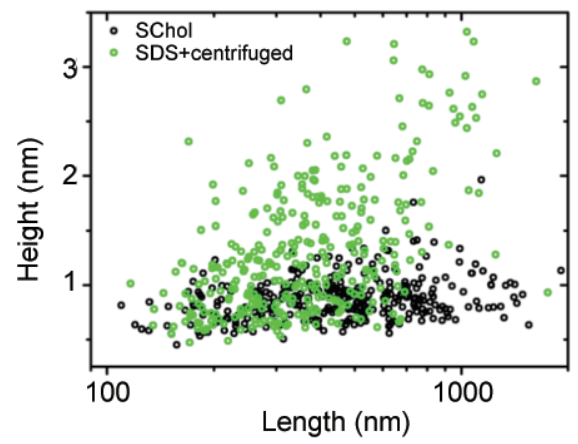

(b)

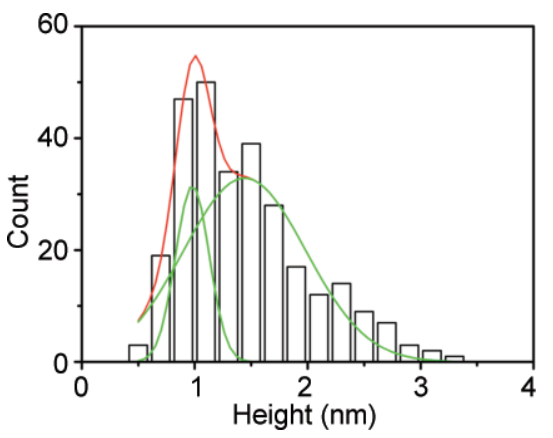

(c)

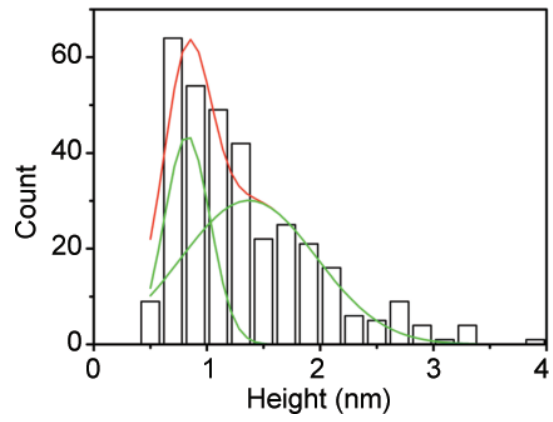

(d)

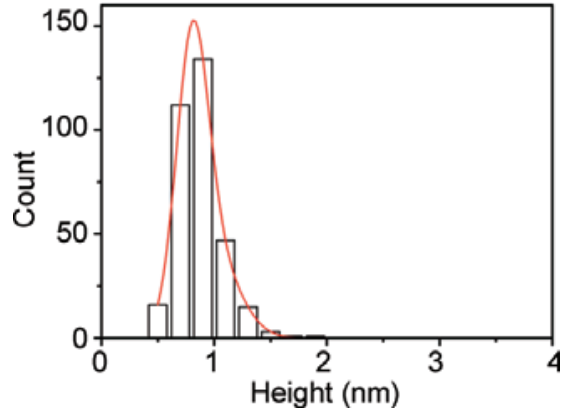

(e)

Figure 2 Length and height distributions of the nanotube material dispersed in the starting SWNT SDS/D ${ }_{2} \mathrm{O}$ suspension used for separation. (a) Sonicated in 1 wt\% SDS and purified with DGC (red) and (b) sonicated in 1 wt\% SDS and centrifuged with out iodixanol (green). Also, shown for comparison are analogous distributions obtained for a typical SChol suspension which yield predominantly individual SWNTs (black)—see text for preparation details. Data were obtained from an analysis of intermittent contact mode AFM images (circles correspond to individual objects). (c) Height distribution of SDS suspension purified by DGC. (d) Height distributions of SDS suspension purified by centrifugation only. (e) Height distribution of the $S C h o l / D_{2} \mathrm{O}$ suspension 
iodixanol (Fig. 2(d)). We therefore conclude that the SDS suspension contained a significant amount of bundles. In contrast, for the SChol suspension we observed (Fig. 2(e)) that most of the measured objects were near $1 \mathrm{~nm}$ in height, suggesting a significantly smaller bundle content. Note that small bundles of two or even three tubes might not be distinguishable from individual tubes in AFM because they lie flat on the surface. Therefore, the quantification of overall bundle content is difficult.

Several studies have shown that SDSin comparison to other commercially available surfactants-is not a particularly good surfactant for the generation of individualized SWNTs in aqueous dispersion. For example, Islam and coworkers analyzed diameter distributions of SWNTs in dispersions prepared with several different surfactants by means of AFM (using a procedure similar to that applied here) and estimated that for SDS, only about $16 \% \pm 2 \%$ of the analyzed objects corresponded to suspended individual isolated tubes [7].

Figures 2(a) and 2(b) also indicate that the SDS starting suspension exhibited a pronounced correlation between object height and length which was not seen in the case of the SChol suspension. Generally, the objects with heights $\leqslant 1 \mathrm{~nm}$, which we assign to individual tubes, are also the shorter ones with lengths $\leqslant 300 \mathrm{~nm}$. Objects longer than 300 $\mathrm{nm}$ preferentially have heights which are larger than $1 \mathrm{~nm}$. From this we infer that in the SDS starting suspension, bundled tubes are typically longer than individualized SWNTs.

From the work of Doorn et al., it is wellestablished that capillary EP can be used to separate bundled SWNTs from isolated individual SWNTs when suspended in SDS $/ \mathrm{D}_{2} \mathrm{O}$. This observation was attributed to differences in nanotube mobility "depending on changes in diameter or bundle size" [8]. Assuming then, that with gel EP we are sorting tubes by length and bundle size, the simplest explanation why EP also achieves separation by electronic property is that after sonication in $\mathrm{D}_{2} \mathrm{O}$ / SDS (and first stage DGC) the SWNT material is already selectively dispersed. In the starting suspension s-SWNTs are present predominantly as small bundles with lengths $\geqslant 300 \mathrm{~nm}$ whereas
m-SWNTs are found primarily as individual tubes having significantly shorter lengths. We have previously demonstrated that individual SWNTs in surfactant suspensions can be shortened to lengths of $\geqslant 100 \mathrm{~nm}$ by appropriate sonication [6]. The mechanistic picture invoked to explain the corresponding shortening dynamics implies that SWNT bundles would not be as effectively cut under the same conditions.

Therefore, we conclude that under the sonication conditions used to generate the starting SDS $/ \mathrm{D}_{2} \mathrm{O}$ suspensions in the present study: (i) m-SWNTs in SDS $/ \mathrm{D}_{2} \mathrm{O}$ are preferentially suspended as individual tubes whereas s-SWNTs on average remain as small bundles and (ii) individualized m-SWNTs are more effectively shortened than s-SWNT bundles.

\subsection{Separation by DGC}

In the previous section we have inferred that sonication in $\mathrm{D}_{2} \mathrm{O} / \mathrm{SDS}$ leads to enrichment of s-SWNTs in small bundles, whereas m-SWNTs are predominantly dispersed as individualized nanotubes. If this inference from the EP study is correct, it should also be possible to separate the two electronic types by making use of the difference in buoyant densities between bundles and individualized SWNTs. Following this logic, we next carried out DGC separation of a starting suspension using a protocol similar to that in Ref. [2] using iodixanol as density gradient medium: in one case with $1 \mathrm{wt} \%$ SDS and in a second experiment with a $1 \mathrm{wt} \%$ solution of a 3:1 mixture of SDS and SChol added. The photograph in Fig. 3(a) shows the resulting density gradient containing a multi-coloured region indicative of separation. Also, shown in (Fig. 3(b) are UV-vis-NIR spectra as measured for the uppermost (green curve) and lowermost (red curve) fractions cut from this coloured region. The spectra obtained for the upper and lower fractions show clear evidence of enrichment of m-SWNTs and s-SWNTs, respectively, compared to the starting PLV-SWNT suspension. Fractions cut in between (not shown here), show intermediate ratios of $\mathrm{m}$ - and s-SWNTs.

In addition to performing UV-vis-NIR measurements, both fractions shown in Fig. 3 were also immediately investigated by AFM. Figure 4 
shows the corresponding height distributions for uppermost (Fig. 4(a)) and lowermost (Fig. 4(b)) fractions. Clearly, the s-SWNT-containing fraction consists of bundles whereas the m-SWNT-fraction mainly consists of individual tubes. This finding therefore corroborates the inference already made by EP, namely that in SDS $/ \mathrm{D}_{2} \mathrm{O}, \mathrm{m}-\mathrm{SWNT}$ are preferentially suspended as individual tubes whereas s-SWNTs tend to remain in bundles.

Arnold et al. have suggested that their separation of $\mathrm{m}$ - and s-SWNTs in DGC using a mixture of SDS and SChol is driven by how the two surfactants organize around SWNTs having different structures and/or electronic types [2]. Clearly, the two chemically very different surfactant molecules could have strongly varying affinities towards $\mathrm{m}$ - or s-SWNTs, in turn giving rise to dramatic variations in nanotube-surfactant, water-surfactant and/or surfactant-surfactant interactions. As a result, variations in surfactant packing density, orientation, and ionization/hydration would be expected. Our experimental findings are consistent with this general picture. Indeed we find that metallic tubes appear to have a greater affinity towards SDS than do semiconducting ones. Therefore, s-SWNTs remain primarily in bundles. Under our conditions however, we make use of differences in densities which arise primarily from differences in bundle sizes and not as a result of the adsorbed surfactant composition. Our $\mathrm{m} / \mathrm{s}-\mathrm{SWNT}$ separation by DGC also works if carried out using a gradient medium comprising iodixanol with $1 \mathrm{wt} \%$ of SDS instead of using a 3:1 mixture of SDS:SChol.

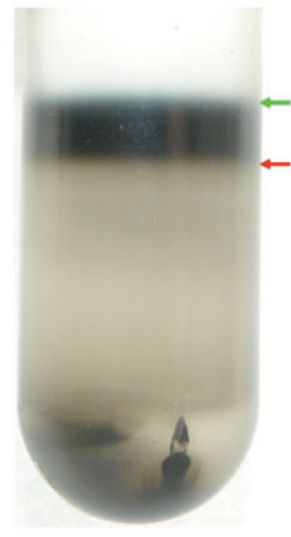

(a)

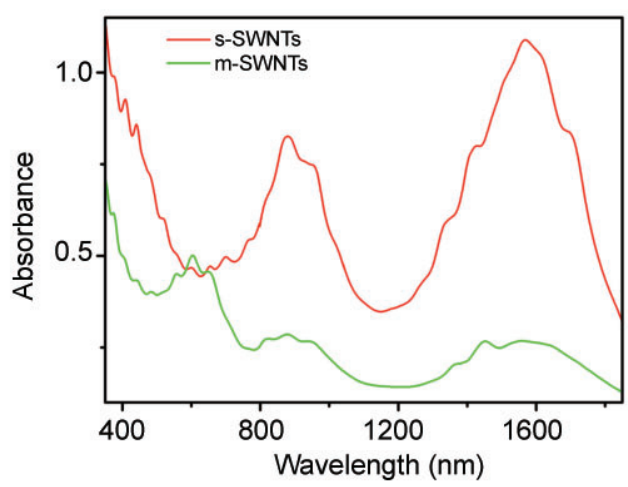

(c)

Figure 3 (a) Photograph taken after DGC separation of the starting suspension using a self-generated gradient of iodixanol plus $1 \mathrm{wt} \%$ of a 3:1 mixture of SDS and SChol; (b) UV-vis-NIR spectra of the starting suspension (black) and of the uppermost (green) and lowermost (red) fractions obtained after DGCas indicated by arrows; (c) UV-vis-NIR spectra of DGC fractions obtained after DGC using a self-generated gradient of iodixanol plus $1 \mathrm{wt} \%$ of SDS only

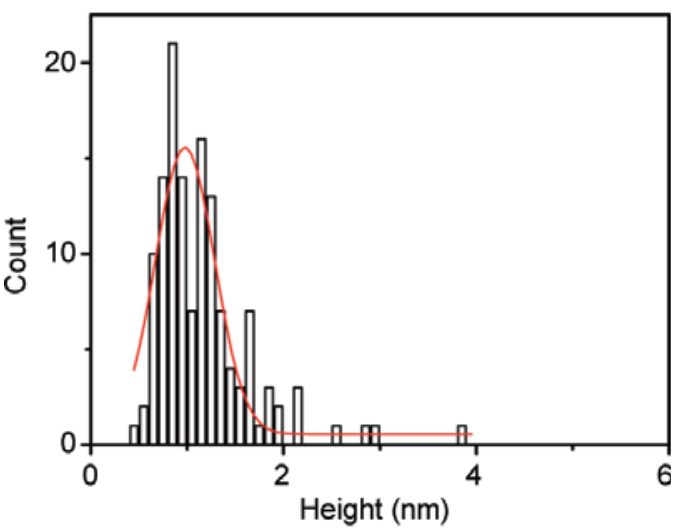

(a)

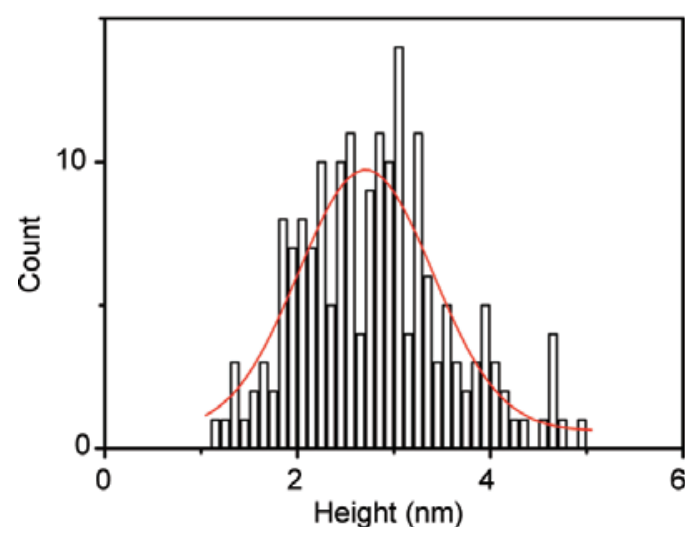

(b)

Figure 4 Height distribution determined by intermittent contact mode AFM for fractions obtained after DGC (see text for details). Shown are data for the uppermost (a) and lowermost (b) fractions 


\subsection{Separation by filtration with a size exclusion chromatography column medium}

Our inferences outlined above suggest that it should also be possible to fractionate the starting suspensions by electronic structure type using simple chromatography techniques like SEC or gel filtration chromatography. SEC separates molecules passing through the column according to differences in their size and has been extensively used to size-separate SWNTs but not for separating $\mathrm{m}$ - from s-SWNTs [6,9-14]. By choosing an appropriate SEC column medium, particle size, gel porosity, and eluant composition we found that it is in fact possible to achieve $\mathrm{m} / \mathrm{s}$-SWNT separation. In this approach we use the SEC column medium as a "filter" to trap the bundles, whereas the smaller individualized SWNTs elute as expected for regular SEC. In particular, we performed the SEC filtration using two different eluants in series. First, the starting suspension was (partly) eluted from the column with $1 \mathrm{wt} \%$ of SDS in water. This led to the rapid elution of the shorter metallic tubes-within the first $50 \mathrm{~mL}$ of eluant applied to the column (Fig. 5(a)). In contrast, the longer, rigid, and bundled semiconducting tubes remained on the column. By subsequently changing the eluant from $1 \mathrm{wt} \%$ SDS in $\mathrm{H}_{2} \mathrm{O}$ to $1 \mathrm{wt} \%$ SChol in $\mathrm{H}_{2} \mathrm{O}$, these trapped bundles were then dissolved and eluted.

The optimum SEC gel found by us for this purpose is specified by the manufacturer to separate molecules with a molar mass in the range of $5 \times 10^{3}-$ $2.5 \times 10^{5} \mathrm{~g} / \mathrm{mol}$. Note that from the AFM study, our individualized SWNTs have an average length of 300 $\mathrm{nm}$ which corresponds to a molar mass of $2 \times 10^{3}-5$ $\times 10^{5} \mathrm{~g} / \mathrm{mol}$ depending on the diameter of the tube. The latter value is already at the upper limit of the fractionation range of the gel. The semiconducting bundles are longer and clearly above this specified molar mass range. Consequently, they are not expected to be able to enter the pores of the SEC medium. Instead, they appear to non-specifically adsorb onto the gel particles. After adding 1\% SChol in $\mathrm{H}_{2} \mathrm{O}$ they become partly dissolved and start to move through the gel. Figure 5(b) shows UV-vis-NIR spectra of SEC fractions obtained by using an eluant of $1 \mathrm{wt} \%$ of SDS in $\mathrm{H}_{2} \mathrm{O}$ (red) followed by an eluant

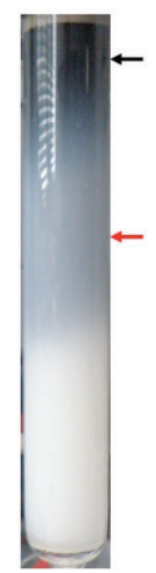

(a)

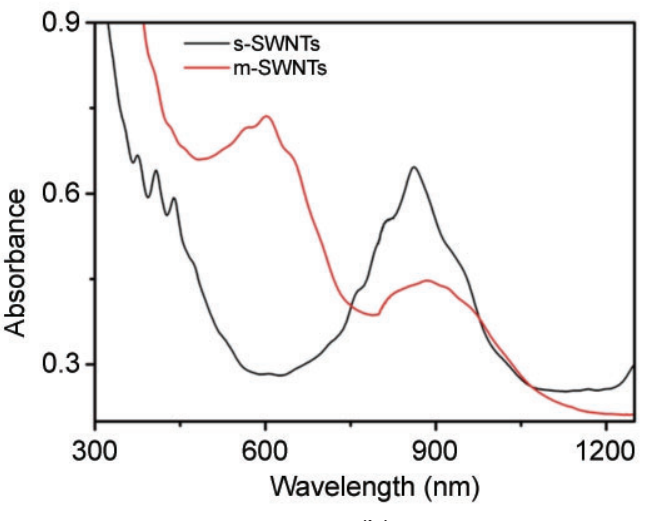

(b)
Figure 5 (a) Photograph taken after adding of the starting suspension to the SEC medium. The m-SWNTs immediately start to propagate through the SEC medium whereas the s-SWNTs become stuck within the first few $\mathrm{cm}$ (indicated by red and black arrows, respectively); (b) UV-Vis-NIR spectra of the eluted SEC fractions obtained after column separation of the starting suspension using first an eluant of $1 \mathrm{wt} \%$ of SDS in water (red) and subsequently an eluant of $1 \mathrm{wt} \%$ of SChol (black)

of 1 wt \% of SChol in $\mathrm{H}_{2} \mathrm{O}$ (black). The m/s-SWNT separation is apparent.

A possible explanation of why SDS is more strongly adsorbed onto m-SWNTs than s-SWNTs can be suggested by analogy to what was proposed by Maeda and co-workers [15]. They demonstrated a simple separation method involving dispersioncentrifugation of HiPco SWNTs using tetrahydrofuran (THF) solutions of various alkylamines. This results in highly concentrated (>87\%) solutions of m-SWNTs. Apparently, the amine/m-SWNT complexes have a higher solubility in THF than in the case for amine/s-SWNTs. Density functional theory (DFT) calculations indicate that the amine alkyl groups interact more strongly with m-SWNTs.

\section{Conclusions}

We have demonstrated that EP-, DGC- and SECbased separation procedures for separating bulk scale samples of metallic and semiconducting SWNTs all rely on starting suspensions in which SWNT dispersion is already selective with respect to electronic structure type. Specifically, we find that the starting SDS $/ \mathrm{D}_{2} \mathrm{O}$ suspensions contain long bundles comprising mainly s-SWNTs as well as shorter 
individualized tubes consisting of predominantly m-SWNTs. DGC then separates by differences in buoyant densities between suspended bundles and individual tubes. Gel EP and SEC filtration separate by differences in mobility between longer/thicker and shorter/thinner objects. With SEC filtration, upscaling should be possible because complex technical processes are not necessary. In future work it will be of interest to simulate and quantify the corresponding SDS-(s/m-)SWNT interactions.

We have recently found that a prefiltration step using a short (disposable) section of SEC column medium can be used instead of 70,000 $\mathrm{g}$ centrifugation to generate starting suspension from the initially dispersed SWNT material. This can then be separated by SEC filtration as described in the text, thus further simplifying the overall procedure.

\section{Acknowledgements}

This research was supported by the Bundesministerium für Bildung und Forschung (BMBF) and by the Deutsche Forschungsgemeinschaft (DFG).

\section{References}

[1] Krupke, R.; Hennrich, F.; von Loehneysen, H.; Kappes, M. $M$. Separation of metallic from semiconducting singlewalled carbon nanotubes. Science 2003, 301, 344-347.

[2] Arnold, M. S.; Green, A. A.; Hulvat, J. F.; Stupp, S. I.; Hersam, M. C. Sorting carbon nanotubes by electronic structure using density differentiation. Nat. Nanotechnol. 2006, 1, 60-65.

[3] Tanaka, T.; Jin, H. H.; Miyata, Y.; Kataura, H. High-yield separation of metallic and semiconducting single-wall carbon nanotubes by agarose gel electrophoresis. Appl. Phys. Express 2008, 1, 114001.

[4] Hersam, M. C. Progress towards monodisperse singlewalled carbon nanotubes. Nat. Nanotechnol. 2008, 3, 387-394.

[5] Lebedkin, S.; Schweiss, P.; Renker, B.; Malik, S.; Hennrich, F.; Neumaier, M.; Stoermer, C.; Kappes, M. M. Singlewall carbon nanotubes with diameters approaching 6 $\mathrm{nm}$ obtained by laser vaporization. Carbon 2002, 40, 417-423.
[6] Hennrich, F.; Krupke, R.; Arnold, K.; Rojas Stuetz, J. A.; Lebedkin, S.; Koch, T.; Schimmel, T.; Kappes, M. M. The mechanism of cavitation-induced scission of singlewalled carbon nanotubes. J. Phys. Chem. B 2007, 111, 1932-1937.

[7] Islam, M. F.; Rojas, E.; Bergey, D. M.; Johnson, A. T.; Yodh. A. G. High weight fraction surfactant solubilization of single-wall carbon nanotubes in water. Nano Lett. 2003, 3, 269-273.

[8] Doorn, S. K.; Strano, M. S.; O'Connell, M. J.; Haroz, E. H.; Rialon, K. L.; Hauge, R. H.; Smalley, R. E. Capillary electrophoresis separations of bundled and individual carbon nanotubes. J. Phys. Chem. B 2003, 107, 60636069.

[9] Duesberg, G. S.; Muster, J.; Krstic, V.; Burghard, M.; Roth, S. Chromatographic size separation of single-wall carbon nanotubes. Appl. Phys. A 1998, 67, 117-119.

[10] Farkas, E.; Anderson, M. E.; Chen, Z. H.; Rinzler, A. G. Length sorting cut single wall carbon nanotubes by high performance liquid chromatography. Chem. Phys. Lett. 2002, 363, 111-116.

[11] Heller, D. A.; Mayrhofer, R. M.; Baik, S.; Grinkova, Y. V.; Usrey, M. L.; Strano, M. S. Concomitant length and diameter separation of single-walled carbon nanotubes. J. Am. Chem. Soc. 2004, 126, 14567-14573.

[12] Huang, X. Y.; McLean, R. S.; Zheng, M. High-resolution length sorting and purification of DNA-wrapped carbon nanotubes by size-exclusion chromatography. Anal. Chem. 2005, 77, 6225-6228.

[13] Yang, Y. L.; Xie, L. M.; Chen, Z.; Liu, M. H.; Zhu, T.; Liu, Z. F. Purification and length separation of single-walled carbon nanotubes using chromatographic method. Synth. Met. 2005, 155, 455-460.

[14] Bauer, B. J.; Fagan, J. A.; Hobbie, E. K.; Chun, J.; Bajpai, V. Chromatographic fractionation of SWNT/DNA dispersions with on-line multi-angle light scattering. J. Phys. Chem. C 2008, 112, 1842-1850.

[15] Maeda, Y.; Kimura, S. -I.; Kanda, M.; Hirashima, Y.; Hasegawa, T.; Wakahara, T.; Lian, Y.; Nakahodo, T.; Tsuchiya, T.; Akasaka, T.; Lu, J.; Zhang, X.; Gao, Z.; Yu, Y.; Nagase, S.; Kazaoui, S.; Minami, N.; Shimizu, T.; Tokumoto, H.; Saito, R. Large-scale separation of metallic and semiconducting single-walled carbon nanotubes. J. Am. Chem. Soc. 2005, 127, 10287-10290. 